\title{
Isotropic-nematic phase diagram for interacting rigid rods on two-dimensional lattices
}

\author{
P. Longone, M. Dávila, and A. J. Ramirez-Pastor ${ }^{*}$ \\ Departamento de Física, Instituto de Física Aplicada, Universidad Nacional de San Luis-CONICET, Chacabuco 917, \\ D5700BWS San Luis, Argentina
}

(Received 3 October 2011; revised manuscript received 23 December 2011; published 20 January 2012)

\begin{abstract}
The phase behavior of interacting rigid rods of length $k$ ( $k$-mers) on two-dimensional square and triangular lattices has been studied by theoretical calculations in the framework of the lattice-gas model. The process was analyzed by comparing the dependence on coverage of the free energy per site of an isotropic submonolayer of interacting $k$-mers $f_{\text {iso }}(\theta)$ with that corresponding to a fully aligned (nematic) system $f_{\text {nem }}(\theta)$. The existence of an intersection point between the curves $f_{\text {iso }}(\theta)$ and $f_{\text {nem }}(\theta)$, which is indicative of the occurrence of an isotropic-nematic phase transition in the adlayer, allowed us to obtain the complete (temperature, coverage, $k$-mer size) phase diagram of the system.
\end{abstract}

DOI: 10.1103/PhysRevE.85.011136

PACS number(s): 05.50.+q, 64.70.mf, 64.60.De

\section{INTRODUCTION}

The study of systems of hard nonspherical colloidal particles has been an attractive and important topic in statistical physics for a long time. An early seminal contribution to this subject was made by Onsager [1], who predicted that very long and thin rods interacting with only excluded volume interaction can lead to long-range orientational (nematic) order. This nematic phase, characterized by a big domain of parallel molecules, is separated from an isotropic state by an isotropic-nematic (IN) phase transition occurring at a finite critical density. Zimm [2] and Isihara [3] also made important contributions to the understanding of the statistics of rigid rods in a dilute solution.

The treatments in Refs. [1-3] are limited in their application because they are valid for a dilute solution only and not applicable to systems of nonsimple shapes. The Flory-Huggins (FH) theory, due independently to Flory [4] and to Huggins [5], has overcome the restriction to a dilute solution by means of a lattice calculation. The results revealed that the lattice model would also show an IN phase transition as a function of density.

The FH statistics, given for the packing of molecules of arbitrary shape but isotropic distribution, provide a natural foundation onto which the effect of the orientation of the admolecules can be added. Following this line of thought, DiMarzio [6] developed an approximate method of counting the number of ways, $\Omega$, to pack together $N$ linear polymers in $M$ sites, given a definite distribution of shapes for the molecules and a definite distribution (continuous or discrete) of the orientations of each shape. Based on the detailed knowledge of the orientations of the molecules, the various types (nematic, smectic, and cholestic) of ordered phases were argued for and the reasons for their existence were ascertained. In the case of allowing only those orientations for which the molecules fit exactly onto the lattice and for an isotropic distribution of $k$-mers, the value of $\Omega$ reduces to the value obtained previously by Guggenheim [7]. We call this limit the Guggenheim-DiMarzio (GD) approximation.

Beyond the works cited above, in the case of two-dimensional (2D) lattice models, the nature of the

\footnotetext{
*antorami@unsl.edu.ar
}

IN transition was an open problem for several decades. Recently, a system of straight rigid rods of length $k$ on a square lattice, with two allowed orientations, was studied by Monte Carlo (MC) simulations [8]. In Ref. [8], Ghosh and Dhar found strong numerical evidence that the system shows nematic order at intermediate densities for $k \geqslant 7$ and provided a qualitative description of a second phase transition (from a nematic order to a nonnematic state) occurring at a coverage close to 1 . In a series of subsequent papers [9-11], it was shown that (1) the transition from the low-density disordered phase to the intermediate-density ordered phase belongs to the 2D Ising universality class for square lattices and the three-state Potts universality class for honeycomb and triangular lattices $[9,10]$; (2) the minimum value of $k\left(k_{\min }\right)$, which allows the formation of a nematic phase, is $k_{\min }=7$ for triangular lattices [11] and $k_{\min }=11$ for honeycomb lattices [10]; and (3) the critical density characterizing the IN transition $\theta_{c}$ follows a power law as $\theta_{c}(k) \propto k^{-1}[11]$ (this dependence was already noted in Ref. [8]).

More recently, the dependence of the critical density on the magnitude of the lateral interactions $w$ was studied for a system of attractive rigid rods on square lattices with two allowed orientations [12,13]. The obtained results in Ref. [12] revealed that the orientational order survives in a wide range of lateral interactions, and that for a fixed value of $k$, the critical density shifts to higher values as the magnitude of the lateral interactions is increased. In addition, the exhaustive MC study in Refs. [12,13] determined that the IN transition does not change the universality class with respect to the case of noninteracting rods.

In the case of noninteracting rods [8-11], MC results have been backed up by theoretical analysis based on entropy considerations [11,14], geometrical arguments [11], a free energy minimization criterion [15], and calculations on a Bethe-like lattice [16]. The same has not happened in the case of interacting rods and, consequently, the IN phase transition predicted by MC techniques in Refs. [12,13] has not been corroborated yet by analytical methods. In this context, the main objective of the present work, using an analytical technique, is to obtain evidence of the existence (or not) of nematic order for appropriate values of $k$ and $w$. The study is based on a comparison between the dependence on coverage of the free energy per site of an isotropic submonolayer of 
interacting $k$-mers $f_{\text {iso }}(\theta)$ and that corresponding to a fully aligned (nematic) system $f_{\text {nem }}(\theta)$. The intersection point of the curves $f_{\text {iso }}(\theta)$ and $f_{\text {nem }}(\theta)$ is indicative of an IN transition and allows us to estimate, from the different approximations used to calculate $f_{\text {iso }}(\theta)$ and $f_{\text {nem }}(\theta)$, the value of coverage at which the phase transition takes place.

The rest of the paper is organized as follows. In Sec. II, the theoretical formalism is presented. The analysis of the results and discussion are given in Sec. III. Finally, the conclusions are drawn in Sec. IV.

\section{MODEL AND THEORY}

Let us assume a system of $N$ interacting linear rigid $k$ mers adsorbed on a lattice of $M$ sites and connectivity $c$. The adsorbate molecules contain $k$ identical units and each one occupies a lattice site. Small adsorbates with spherical symmetry would correspond to the monomer limit $(k=1)$. The distance between $k$-mer units is assumed in registry with the lattice constant $a$; hence, exactly $k$ sites are occupied by a $k$-mer when adsorbed. Two different energies are considered in the adsorption process: (1) $U_{0}$, the constant interaction energy between a $k$-mer unit and an adsorption site, and (2) $w$, the lateral interaction energy between two nearest-neighbor (NN) units belonging to different $k$-mers, which is assumed to be either repulsive (positive) or attractive (negative). Under these conditions, the Hamiltonian of the system can be written as

$$
H=w \sum_{\langle i, j\rangle} c_{i} c_{j}-N(k-1) w+\left(U_{o}-\mu\right) \sum_{i} c_{i},
$$

where $\mu$ is the chemical potential, $\langle i, j\rangle$ represents pairs of NN sites, and $c_{i}$ is the occupation variable, which can take the following values: $c_{i}=0$ if the corresponding site is empty and $c_{i}=1$ if the site is occupied. The term $N(k-1) w$ is subtracted in Eq. (1) since the summation over all of the pairs of $\mathrm{NN}$ sites overestimates the total energy by including $N(k-1)$ bonds belonging to the $N$ adsorbed $k$-mers.

As it was established in Refs. [12,13], a continuous IN phase transition occurs in the system at an intermediate density for appropriate values of the $k$-mer size and the magnitude of the lateral interaction. The simulation data show interesting temperature-coverage phase diagrams, where the critical lines (separating isotropic and nematic regions) depend on $k$ and $w$. In what follows, we will try to obtain a theoretical confirmation of this phase behavior. The procedure chosen is based on a comparison between the dependence on coverage of the free energy per site of an isotropic adlayer of attractive $k$-mers $f_{\text {iso }}(\theta)$ and that corresponding to a fully aligned system (nematic phase) $f_{\text {nem }}(\theta)$. As it will be shown below, the condition $f_{\text {iso }}(\theta)-f_{\text {nem }}(\theta)=0$ (intersection point of the curves $f_{\text {iso }}$ and $f_{\text {nem }}$ ) is indicative of an IN transition. This property allows us to estimate the value of coverage at which the phase transition takes place and, consequently, to obtain the complete phase diagram of the system.

For the isotropic system, an analytical expression for the free energy per site was obtained explicitly in Refs. [17], using the configuration-counting procedure of the quasichemical approach (QCA),

$$
\begin{aligned}
\beta f_{\text {iso }}(\theta)= & -\frac{\theta}{k} \ln q+\beta \frac{\theta}{k}\left(\frac{\lambda w}{2}+k U_{0}\right)-\lambda \frac{\theta}{k} \ln \frac{\lambda \theta}{c k}+\left[c-2\left(\frac{k-1}{k}\right) \theta\right] \ln \left[1-\frac{2}{c}\left(\frac{k-1}{k}\right) \theta\right]-\left[\frac{c}{2}-\left(\frac{k-1}{k}\right) \theta\right] \\
& \times \ln \left[\frac{c}{2}-\left(\frac{k-1}{k}\right) \theta\right]+\frac{\lambda \theta}{2 k} \ln \left(\frac{\lambda \theta}{2 k}-\alpha\right)-c\left[1-\frac{2}{c}\left(\frac{k-1}{k}\right) \theta-\frac{\lambda \theta}{c k}\right] \ln \left[1-\frac{2}{c}\left(\frac{k-1}{k}\right) \theta-\frac{\lambda \theta}{c k}\right] \\
& +\left[\frac{c}{2}-\left(\frac{k-1}{k}\right) \theta-\frac{\lambda \theta}{2 k}\right] \ln \left[\frac{c}{2}-\left(\frac{k-1}{k}\right) \theta-\frac{\lambda \theta}{2 k}-\alpha\right]-\ln \gamma,
\end{aligned}
$$

where $\beta=1 / k_{B} T$ ( $k_{B}$ is the Boltzmann constant), $\alpha$ is

$$
\begin{gathered}
\alpha=\frac{\lambda c}{2 k} \frac{\theta(1-\theta)}{\left[\frac{c}{2}-\left(\frac{k-1}{k}\right) \theta+b\right]}, \\
b=\left\{\left[\frac{c}{2}-\left(\frac{k-1}{k}\right) \theta\right]^{2}-\frac{\lambda c}{k} A \theta(1-\theta)\right\}^{1 / 2},
\end{gathered}
$$

and

$$
\gamma=\Omega(N, M)^{1 / M} .
$$

$\Omega(N, M)$ is the number of ways to arrange $N k$-mers on $M$ sites. In general, $\Omega(N, M)$ depends on the spatial configuration (shape) of the $k$-mer and the surface geometry. Even in the simplest case of dimers, the exact form of $\Omega(N, M)$ in two (or more) dimensions does not exist. However, as discussed in Sec. I, different approximations have been developed for
$\Omega(N, M)$. In this paper, three theories have been considered: the first is the well-known FH approximation [4,5], the second is the GD approach for rigid rod molecules [6,7], and the third is the recently developed semiempirical (SE) model for adsorption of polyatomics [18]. The corresponding expressions for the factor $\gamma$ are as follows:

$$
\begin{gathered}
\ln \gamma(\theta)=-\frac{\theta}{k} \ln \frac{\theta}{k}-(1-\theta) \ln (1-\theta) \\
-\frac{\theta}{k}\left[k-1-\ln \left(\frac{c}{2}\right)\right] \quad(\mathrm{FH}), \\
\ln \gamma(\theta)=-\frac{\theta}{k} \ln \frac{\theta}{k}-(1-\theta) \ln (1-\theta)+\left(\theta-\frac{c}{2}\right) \ln \left(\frac{c}{2}\right) \\
+\left[\frac{c}{2}-\left(\frac{k-1}{k}\right) \theta\right] \ln \left[\frac{c}{2}-\left(\frac{k-1}{k}\right) \theta\right] \quad(\mathrm{GD}),
\end{gathered}
$$


and

$$
\begin{aligned}
\ln \gamma(\theta)= & -\frac{\theta}{k} \ln \frac{\theta}{k}-(1-\theta) \ln (1-\theta)+\theta\left[\frac{1}{2}-\frac{c}{4}+\frac{1}{k} \ln \left(\frac{c}{2}\right)\right]+\frac{1}{2} \frac{k}{(k-1)}\left[1-\frac{(k-1)^{2}}{k^{2}} \theta^{2}\right] \ln \left[1-\frac{(k-1)}{k} \theta\right] \\
& -\frac{c}{4}\left[\theta+\frac{k(c-4)+4}{2(k-1)}\right]\left[1-\frac{2(k-1)}{c k} \theta\right] \ln \left[1-\frac{2(k-1)}{c k} \theta\right] \text { (SE). }
\end{aligned}
$$

We will now analyze the case corresponding to the nematic state. Specifically, the free energy per site of a 2D submonolayer of aligned $k$-mers should be determined. In order to do so, the following assumptions have been made: (i) The entropy per site of the 2D nematic phase $s_{\text {nem }}(\theta)$ can be approximated by the expression of the entropy per site corresponding to a system of interacting $k$-mers adsorbed in $1 \mathrm{D}, s_{1 D}(\theta)$. As in Eq. (2), $s_{1 D}(\theta)$ will be calculated from the QCA, which provides exact results in 1D [19],

$$
\begin{aligned}
\frac{s_{\text {nem }}(\theta)}{k_{B}} \approx & \frac{s_{1 D}(\theta)}{k_{B}}=\frac{\theta}{k} \ln \frac{\theta}{k}+(1-\theta) \ln (1-\theta)-2 \alpha \ln \alpha \\
& -\left(\frac{\theta}{k}-\alpha\right) \ln \left(\frac{\theta}{k}-\alpha\right)-(1-\theta-\alpha) \\
& \times \ln (1-\theta-\alpha)
\end{aligned}
$$

And (ii), the energy per site of the $2 \mathrm{D}$ nematic phase can be written as

$$
u_{\mathrm{nem}}(\theta)=u_{\mathrm{sus}}(\theta)+u_{\|}(\theta)+u_{\perp}(\theta),
$$

where $u_{\text {sus }}(\theta)$ is the adsorbate-substrate interaction energy, $u_{\|}(\theta)$ takes into account the interaction energy between pairs of NN beads (belonging to different rods) adsorbed along the direction of the nematic phase, and $u_{\perp}(\theta)$ represents the interaction energy between pairs of $\mathrm{NN}$ beads (belonging to different rods) arranged transversely to the direction of the nematic phase (see Fig. 1).

The adsorbate-substrate energy per site results in

$$
u_{\mathrm{sus}}(\theta)=\frac{k N U_{0}}{M}=\theta U_{0} .
$$

On the other hand, $u_{\|}(\theta)$ can be obtained from the QCA (with the exact solution corresponding to rigid rods adsorbed in 1D) [19],

$$
u_{\|}(\theta)=w\left(\frac{\theta}{k}-\alpha\right) .
$$

In the case of the transverse couplings, the corresponding energy term can also be calculated from a mean-field approximation [19],

$$
u_{\perp}=\frac{\bar{N}_{11} w}{M},
$$

where $\bar{N}_{11}$ is the mean number of pairs of NN units (belonging to different $k$-mers) arranged transversely to the direction of the nematic phase. $\bar{N}_{11}$ can be obtained as the product of the number of adsorbed rods $(N)$, the number of $\mathrm{NN}$ pairs in the direction transverse to the axis of a $\operatorname{rod}[\lambda=(c-2) k]$, the mean coverage of the lattice $(\theta=k N / M)$, and a factor of $1 / 2$ to avoid double counting. Then,

$$
u_{\perp}=\frac{1}{2} N[(c-2) k]\left(\frac{k N}{M}\right)\left(\frac{w}{M}\right)=\frac{1}{2}(c-2) w \theta^{2} .
$$

Finally, from Eqs. (2)-(14), the free energy per site of the nematic phase can be written as

$$
\begin{aligned}
\beta f_{\text {nem }}(\theta)= & \beta U_{0} \theta+\beta w\left(\frac{\theta}{k}-\alpha\right)+\beta \frac{1}{2}(c-2) w \theta^{2}-\frac{\theta}{k} \ln \frac{\theta}{k} \\
& -(1-\theta) \ln (1-\theta)+2 \alpha \ln \alpha+\left(\frac{\theta}{k}-\alpha\right) \\
& \times \ln \left(\frac{\theta}{k}-\alpha\right)+(1-\theta-\alpha) \ln (1-\theta-\alpha) .
\end{aligned}
$$

\section{RESULTS}

\section{A. Comparison between simulation and theoretical results}

The reaches and limitations of $f_{\text {iso }}(\theta)$ [Eq. (2)] have been widely discussed in Refs. [17]. In the case of $f_{\text {nem }}(\theta)$, grand canonical MC simulations [20,21] were performed in the present work in order to test the validity of Eq. (15). The MC procedure used is as follows. For a given value of the temperature $T$ and chemical potential $\mu$, an initial nematic configuration with $N$ rigid $k$-mers adsorbed along one of the lattice directions is generated. Then, an adsorptiondesorption process is started, where a linear $k$-uple of $\mathrm{NN}$ sites (located along the direction of the nematic alignment) is chosen at random and an attempt is made to change its occupancy state with probability given by the Metropolis rule, $P=\min [1, \exp (-\beta \Delta H)][22]$, where $\Delta H=H_{f}-H_{i}$ is the difference between the Hamiltonians of the final and initial states. A Monte Carlo step (MCS) is achieved when $M k$-uples of sites have been tested to change their occupancy state. The equilibrium state can be well reproduced after discarding the first $m_{0}=10^{7} \mathrm{MCS}$. Then, averages are taken over $m=10^{7}$ successive configurations.

In our MC simulations, we varied the chemical potential $\mu$ and monitored the density $\theta=k\langle N\rangle / M$, where $\langle\cdots\rangle$ means the average over the $m \mathrm{MC}$ simulation runs. Once $\mu(\theta)$ was obtained, the free energy per site was calculated by using the thermodynamic integration method [23]. The method in the grand canonical ensemble relies on the integration of the chemical potential $\mu$ on coverage along a reversible path between an arbitrary reference state and the desired state of the system. Thus, for $N$ particles on $M$ lattice sites,

$$
F(N, M, T)=F\left(N_{0}, M, T\right)+\int_{N_{0}}^{N} \mu d N^{\prime} .
$$




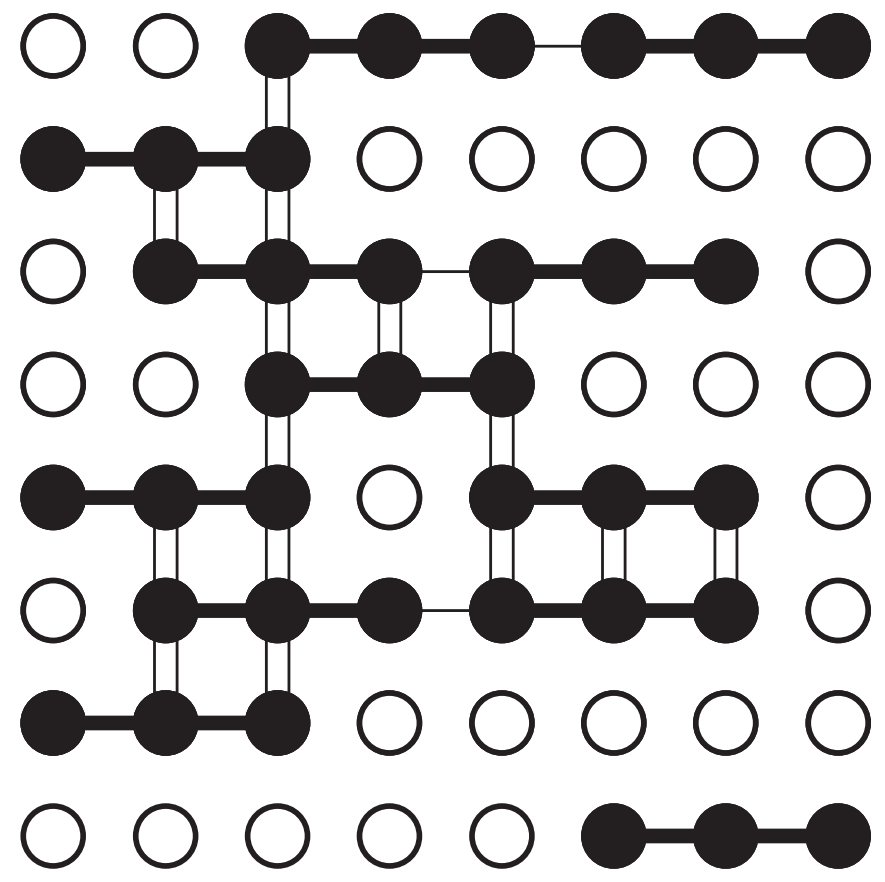

FIG. 1. Nematic phase of trimers adsorbed on a square lattice. Solid circles (joined by thick lines) and empty circles represent trimers and empty sites, respectively. Parallel (transverse) couplings are denoted by single (double) lines.

In our case, the determination of the free energy in the reference state, $F\left(N_{0}, M, T\right)$, is trivial $\left[F\left(N_{0}, M, T\right)=0\right.$ for $\left.N_{0}=0\right]$. Note that the reference state, $N \rightarrow 0$, is obtained for $\mu / k_{B} T \rightarrow-\infty$. Finally, Eq. (16) can be written in terms of intensive variables,

$$
f(\theta, T)=\int_{0}^{\theta} \frac{\mu\left(\theta^{\prime}\right)}{k} d \theta^{\prime} .
$$

An extensive comparison between theoretical results from Eq. (15) (lines) and thermodynamic integration data (symbols) for various $k$-mer sizes and interaction energies is shown in Fig. 2. The general features of the coverage dependence of $\beta f_{\text {nem }}$ are the following. In the limit $\theta \rightarrow 0$, the free energy tends to zero. For low densities, $\beta f_{\text {nem }}$ is a decreasing function of $\theta$; it reaches a minimum, then increases monotonically to a finite value for $\theta=1$. In all cases, the agreement between theory and the MC simulation is excellent, which validates the use of Eq. (15) in the range of interaction energies studied. As will be seen in the next section, this is the range of interest in this paper.

\section{B. Isotropic-nematic phase diagram}

As discussed in Sec. II A, the critical properties of the system have been studied by comparing the curves of $f_{\text {iso }}(\theta)$ [Eq. (2)] and $f_{\text {nem }}(\theta)$ [Eq. (15)] at fixed values of $k$ and $w / k_{B} T$. This strategy has been successfully used in previous works $[8,10,11,14]$. Based on the comparison between the configurational entropy of an isotropic system and that corresponding to a fully aligned system, Ghosh and Dhar found convincing evidence of the existence of a reentrant phase transition from a nematic phase to a disordered phase for a system of noninteracting straight rigid rods at concentrations close to

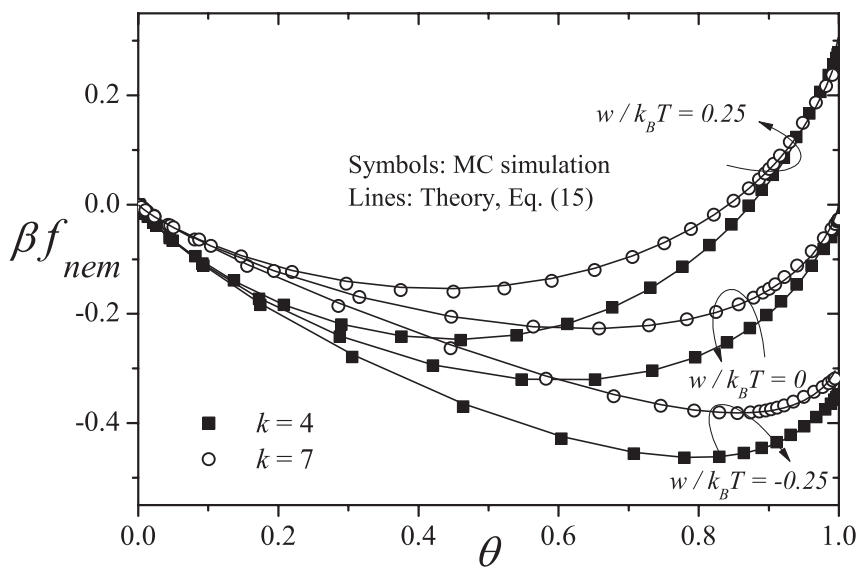

FIG. 2. Nematic free energy per site (in units of $k_{B} T$ ) vs surface coverage for various $k$-mer sizes and interaction energies as indicated and $L / k=20$. Symbols represent MC data and solid lines correspond to theoretical results from Eq. (15).

1 [8]. Later, a similar technique was used in Refs. [10,11,14] to obtain the dependence on $k$ of the coverage at which the IN phase transition takes place in the same system studied by Ghosh and Dhar [8], that is, $\theta_{c}(k) \propto k^{-1}$. In both cases, the technique provided valuable information on the phase transition occurring in the system. However, the method does not allow for a characterization of the nature of the phase transition; in particular, the determination of whether it is first order or continuous is out of reach for this approach.

A typical example of the procedure described in the preceding paragraph is shown in Fig. 3. In this case, $k=7$, $w / k_{B} T=0.25$, and the curve corresponding to the isotropic state was obtained using the GD configurational factor $\gamma$. The isotropic (dashed line) and nematic (solid line) curves cross at an intermediate density (in this case, $\theta=0.7705$ ), and two well-differentiated regimes can be observed. In the first regime, which occurs at low densities, the nematic approach predicts a larger free energy than the isotropic data. In the second regime (at high densities), the behavior is inverted and the nematic data present a smaller free energy than the isotropic results. The crossing of the curves shows that at high densities, it is more favorable for the rods to align spontaneously, since the resulting loss of orientational entropy is by far compensated by the gain of translational entropy. Thus, the condition $f_{\text {iso }}(\theta)-f_{\text {nem }}(\theta)=0$ is indicative of an IN transition. In addition, the intersection of the free energies with different slopes seems to indicate a first-order transition. However, as indicated above, the methodology in Fig. 3 does not allow us to be conclusive about the order of the phase transition occurring in the system [24].

By repeating the procedure of Fig. 3 for different values of $w / k_{B} T$, the phase diagram of Fig. 4 was obtained. Full circles on the solid line have been calculated from the crossing points between the curves $f_{\text {iso }}(\theta)$ and $f_{\text {nem }}(\theta)$. The line separates regions of isotropic and nematic stability. The different phases are shown schematically in the figure.

By analyzing the curve in Fig. 4, two main features deserve to be highlighted. First, $\theta \rightarrow 1$ as $w / k_{B} T \rightarrow \infty$; this finding indicates that the nematic order disappears for strong repulsive interactions. Second, there exists a limit value of the magnitude 


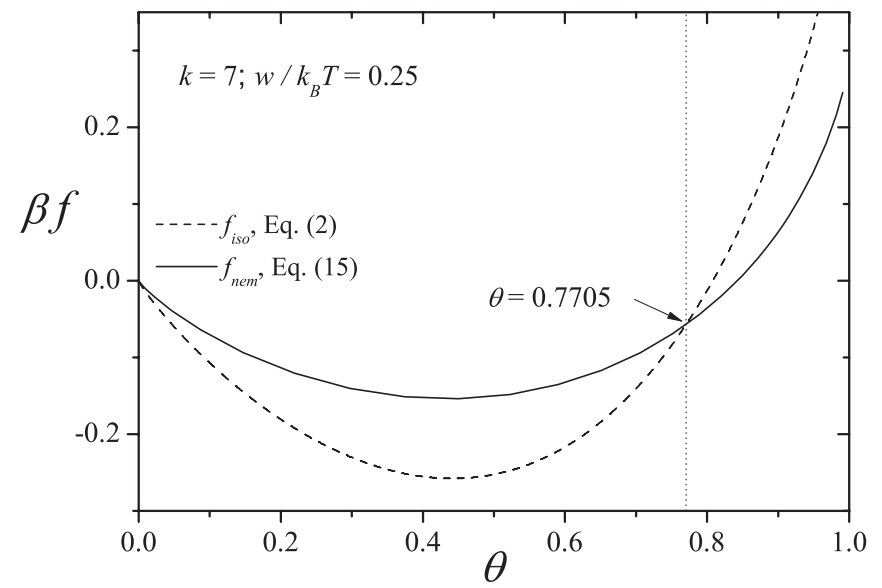

FIG. 3. Comparison between the isotropic [dashed line, Eq. (2)] and nematic [solid line, Eq. (15)] free energies for $k$-mers adsorbed on square lattices with $k=7$ and $w / k_{B} T=0.25$. From their intersections, one obtained the value of the density at which the phase transition takes place. The curve corresponding to the isotropic state was obtained by using the GD configurational factor $\gamma$.

of the lateral interaction energy $w_{l} / k_{B} T$, such that $\theta \rightarrow 0$ as $w / k_{B} T \rightarrow w_{l} / k_{B} T$ (in the case shown in the figure, $\left.w_{l} / k_{B} T=-0.086\right)$, and, consequently, the isotropic phase disappears for $w / k_{B} T<w_{l} / k_{B} T$.

The study of Fig. 4 was extended to a wide range of values of $k$. In this way, a complete $\left(\theta, k, w / k_{B} T\right)$ isotropic-nematic phase diagram was obtained (see Fig. 5). As discussed above, the region below the boundary surface (plotted in gray color) corresponds to the isotropic region, and the region above the boundary surface corresponds to the nematic region. The main characteristics of the phase diagram in Fig. 5 will be discussed below.

We start analyzing the intersection of the boundary surface with the plane $\theta=0$. The resulting curve, $w_{l} / k_{B} T$ vs $k$, is

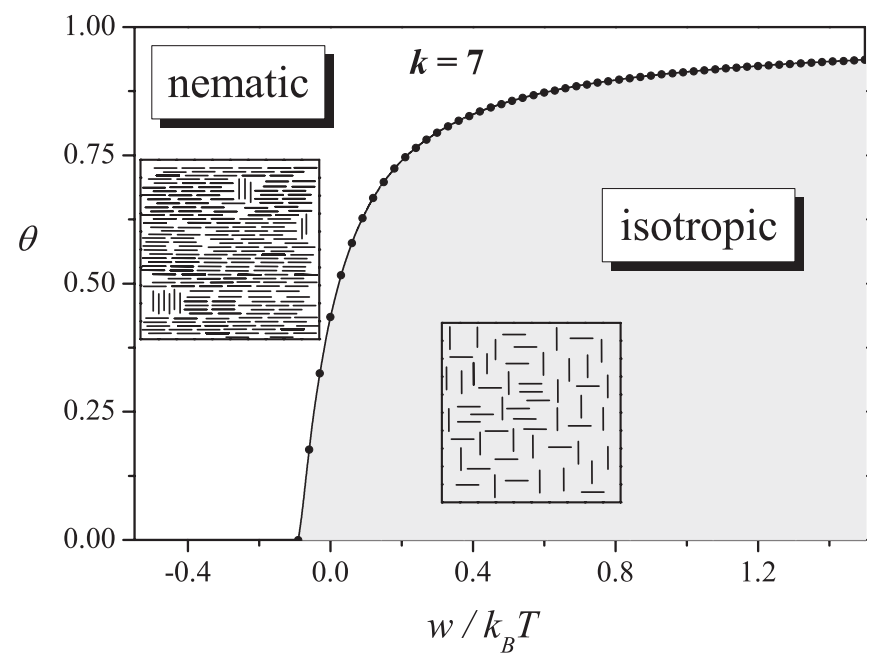

FIG. 4. Isotropic-nematic phase diagram for 7-mers adsorbed on square lattices. The boundary line (solid circles and solid line) separates regions of isotropic and nematic stability. The different phases are shown schematically in the figure. As in Fig. 3, the calculations corresponding to the isotropic state were done by using the GD approximation.

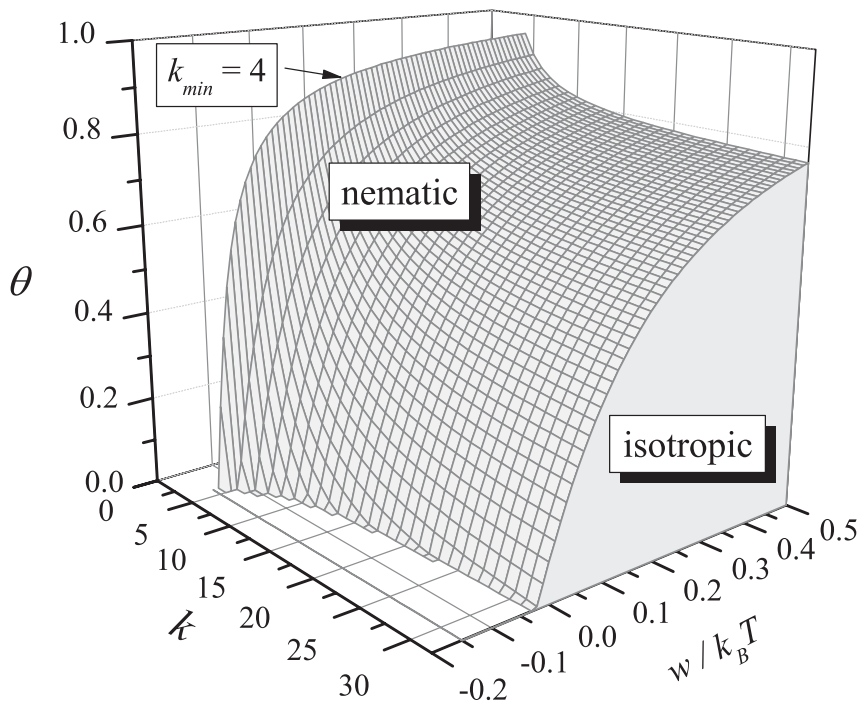

FIG. 5. Isotropic-nematic phase diagram (in the $\theta, k, w / k_{B} T$ parameter space) corresponding to interacting rigid rods adsorbed on square lattices. As in Figs. 3 and 4, the calculations corresponding to the isotropic state were done by using the GD approximation.

shown in Fig. 6. Circles (squares) [triangles] correspond to calculations obtained by using the GD (FH) [SE] configurational factor $\gamma$. For a fixed size $k$, two different regimes can be distinguished according to the value of the lateral interaction energy. For $w / k_{B} T>w_{l} / k_{B} T$, an isotropic-nematic phase transition occurs in the system at an intermediate density. For $w / k_{B} T<w_{l} / k_{B} T$, no phase transition occurs (or the transition density is zero) and the system presents nematic order in all ranges of coverage $(0 \leqslant \theta \leqslant 1)$. As $k$ is increased, $w_{l} / k_{B} T$ tends monotonically to zero. Thus, for large values of $k(k \gtrsim 100)$, the IN phase transition only exists for repulsive lateral interactions. In the case of large $k$-mers and attractive interactions, the coverage at which the phase transition takes place tends to zero and the nematic order prevails for all density.

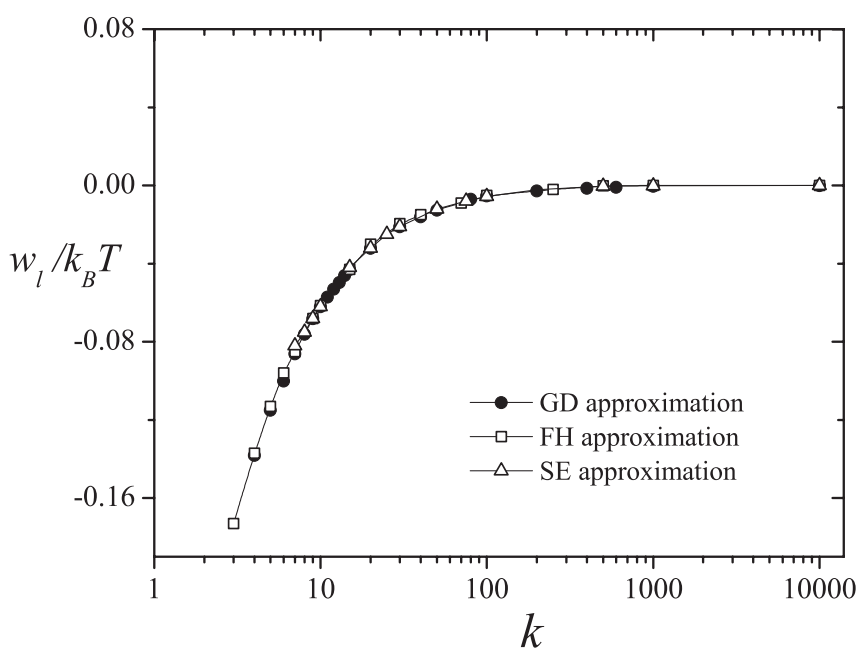

FIG. 6. Dependence of $w_{l} / k_{B} T$ (as defined in the text) on $k$ for $c=4$ (square lattices) and different expressions of the configurational factor $\gamma$ : GD approximation, circles; FH approximation, squares; and SE approximation, triangles. 


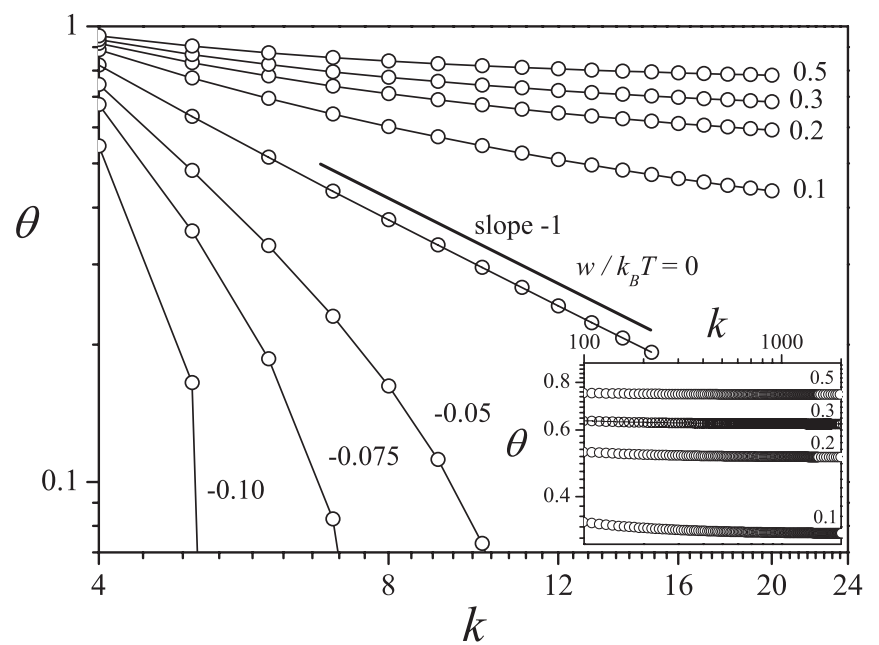

FIG. 7. Log-log plots of the dependence of the phase transition density on $k$ for square lattices, $\gamma$, from Eq. (7) and different values of $w / k_{B} T$, as indicated.

To complete the analysis of Fig. 6, it is important to note that the data of $w_{l} / k_{B} T$ vs $k$ do not depend on the approximation chosen for $\gamma$. This result reinforces our previous conclusion concerning the effect of $k$ and $w / k_{B} T$ on the IN transition occurring in the system.

In previous works $[10,11]$, the dependence of the critical density on the particle size $k$ has been reported for a system of noninteracting rigid rods on two-dimensional lattices, that is, $\theta_{c}(k) \propto k^{-1}$. In addition, the minimum value of $k\left(k_{\min }\right)$, which allows the formation of a nematic phase on a given geometry, was determined. The results were obtained by combining Monte Carlo simulations, finite-size scaling techniques, and theoretical analysis based on entropy considerations. We now ask the following: what happens when lateral interactions are added? To answer this question, let us consider the projection of the boundary surface in Fig. 5 on the $(\theta, k)$ plane. The result of this procedure is shown in Fig. 7. The log-log plots show three different behaviors: (1) For $w / k_{B} T=0$, the phase transition density follows a power law $k^{-1}$, as previously reported $[10,11]$. (2) For repulsive interactions, the transition coverage monotonically decreases as $k$ is increased, asymptotically tending to a finite limit for a large size of adparticles. This limit value increases for increasing values of $w / k_{B} T$. (3) For attractive interactions, the transition density rapidly tends to zero as $k$ is increased.

The limit values obtained in Fig. 7 have been collected in Fig. 8. The resulting curve (thick solid line) represents the isotropic-nematic phase diagram for a system of large straight rigid rods and can be interpreted as the continuum limit of the present model. In other words, the highlighted curve in Fig. 8 represents the phase diagram corresponding to thin needles, with a discrete set of orientations, deposited on a continuum surface. The results indicate that while attractive needles show nematic order in all ranges of coverage, repulsive needles show orientational order only at high values of density. For comparison purposes, the IN phase diagrams corresponding to different finite values of $k$ have been included in Fig. 8.

The behavior obtained for the dependence of the phase transition density on the particle size $k$ from $\mathrm{FH}$ and $\mathrm{SE}$

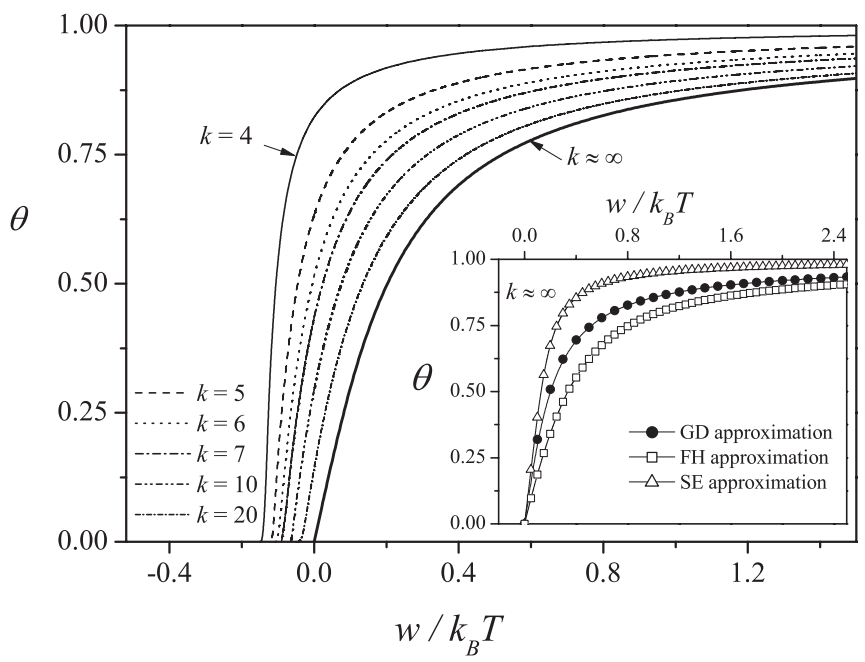

FIG. 8. Isotropic-nematic phase diagrams for $k$-mers adsorbed on square lattices with $\gamma$ from Eq. (7). The different curves correspond to different values of $k$ ranging between $k=4$ and $k \approx \infty$, as indicated. Inset: $\mathrm{FH}, \mathrm{GD}$, and $\mathrm{SE}$ isotropic-nematic phase diagrams for a system of large $(k \approx \infty)$ straight rigid rods on square lattices.

approximations is similar to that reported in Fig. 7. The same occurs for the limit curve $(k \approx \infty)$ shown in Fig. 8 (see inset). With respect to $k_{\min }, \mathrm{FH}$ and $\mathrm{SE}$ predict values of $k_{\min }=3$ and $k_{\min }=7$, respectively, instead of $k_{\min }=4$ as predicted by using the GD approach.

Finally, in order to analyze the effect of the geometry on the phase behavior of the system, the $\left(\theta, k, w / k_{B} T\right)$ IN diagram was also calculated for triangular lattices $(c=6)$. The results obtained (not shown here for brevity) indicate that a very similar behavior is found between the data in Fig. 5 and the corresponding ones for $c=6$. This qualitative agreement can be clearly observed in Fig. 9, where the dependence of $w_{l} / k_{B} T$ on $k$ and the IN phase diagram for large $(k \approx \infty)$ straight

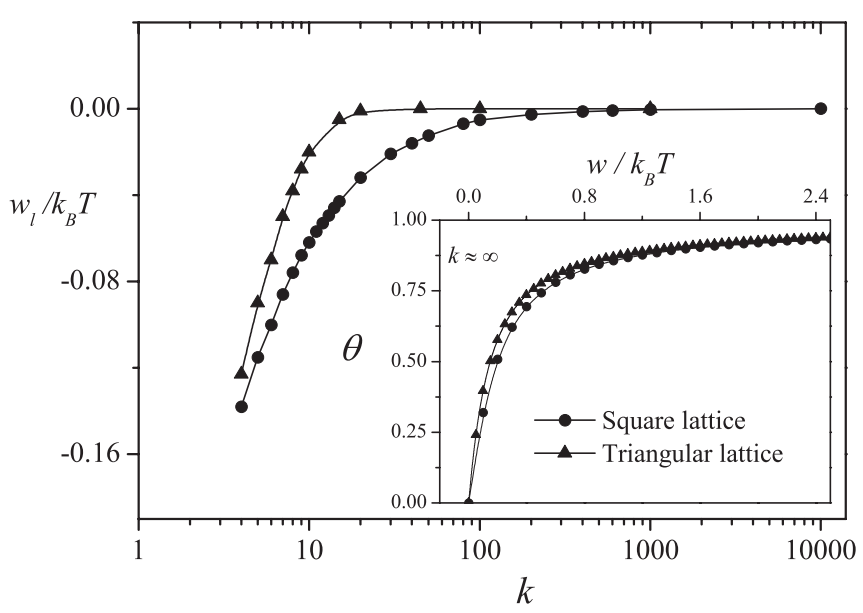

FIG. 9. Dependence of $w_{l} / k_{B} T$ (as defined in the text) on $k$ for square and triangular lattices. Inset: effect of the geometry on the isotropic-nematic phase diagram for large $(k \approx \infty)$ straight rigid rods. In all cases, the calculations corresponding to the isotropic state were done using the GD approximation. 
rigid rods (inset) have been plotted for square and triangular lattices. In both cases, the calculations corresponding to the isotropic state were done using the GD approximation. From a quantitative point of view, the main difference between the two geometries is in the values obtained for the minimum value of $k$ at which the phase transition occurs, namely, $k_{\min }=$ $4(\mathrm{FH}), k_{\min }=4(\mathrm{GD})$, and $k_{\min }=10(\mathrm{SE})$ for triangular lattices.

\section{CONCLUSIONS}

In this paper, the phase behavior of interacting rigid rods of length $k$ on two-dimensional lattices has been addressed. The results were obtained by theoretical analysis, based on the comparison between the dependence on coverage of the free energy per site of an isotropic submonolayer $f_{\text {iso }}(\theta)$ and that corresponding to a fully aligned system (nematic phase) $f_{\text {nem }}(\theta)$. From the intersection point of the curves $f_{\text {iso }}(\theta)$ and $f_{\text {nem }}(\theta)$, a complete $\left(\theta, k, w / k_{B} T\right)$ isotropic-nematic phase diagram was obtained. This diagram is characterized by the following properties:

(a) For a given value of $k$, the transition density tends to 1 as $w / k_{B} T$ tends to $\infty$; this finding indicates that the nematic order disappears for strong repulsive interactions.

(b) For each value of $k$, there exists a limit value of the magnitude of the lateral interaction energy $w_{l} / k_{B} T$, such that the density at which the phase transition takes place tends to 0 as $w / k_{B} T \rightarrow w_{l} / k_{B} T$, and, consequently, the isotropic phase disappears for $w / k_{B} T<w_{l} / k_{B} T$.

(c) As $k$ is increased, $w_{l} / k_{B} T$ tends monotonically to zero and the IN phase transition only exists for repulsive lateral interactions. In the case of large $k$-mers and attractive interactions, the phase transition coverage tends to zero and the nematic order prevails for all density. This limit $(k \rightarrow \infty)$ can be interpreted as the continuum limit of the present model. (d) Three different regimes have been observed for the dependence of the transition density on the particle size $k$ : (1) For $w / k_{B} T=0$, the transition density follows a power law $k^{-1}$, as previously reported $[10,11]$. (2) For repulsive interactions, the transition coverage monotonically decrease as $k$ is increased, asymptotically tending to a finite limit for a large size of adparticles. This limit value of $\theta$ increases for increasing values of $w / k_{B} T$. (3) For attractive interactions, the transition density rapidly tends to zero as $k$ is increased.

(e) The minimum value of $k$, which allows the formation of a nematic phase, depends on the surface geometry and the different theoretical approximations used to calculate $f_{\text {iso }}(\theta)$ and $f_{\text {nem }}(\theta)$, namely, $k_{\min }=3(\mathrm{FH}), k_{\min }=4(\mathrm{GD})$, and $k_{\min }=$ 7 (SE) for square lattices, and $k_{\min }=4(\mathrm{FH}), k_{\min }=4(\mathrm{GD})$, and $k_{\min }=10(\mathrm{SE})$ for triangular lattices.

(f) No qualitative differences were found between the GD, $\mathrm{FH}$, and SE results.

(g) No qualitative differences were observed when the surface geometry was changed.

In summary, the proposed theoretical scheme is simple and confirms the main results obtained in Refs. [12,13] by MC simulation. However, as expected for a mean-field model, the present approach is not able to quantitatively reproduce the $\mathrm{MC}$ data and more efforts are needed for a complete theoretical analysis of the phase behavior of the system.

\section{ACKNOWLEDGMENTS}

This work was supported in part by CONICET (Argentina) under Project No. PIP 112-200801-01332, Universidad Nacional de San Luis (Argentina) under Project No. 322000, and the National Agency of Scientific and Technological Promotion (Argentina) under Project No. PICT-2010-1466.
[1] L. Onsager, Ann. NY Acad. Sci. 51, 627 (1949).

[2] B. H. Zimm, J. Chem. Phys. 14, 164 (1946).

[3] A. Isihara, J. Chem. Phys. 18, 1446 (1950); 19, 1142 (1951).

[4] P. J. Flory, J. Chem. Phys. 10, 51 (1942); Principles of Polymers Chemistry (Cornell University Press, Ithaca, NY, 1953).

[5] M. L. Huggins, J. Phys. Chem. 46, 151 (1942); Ann. NY Acad. Sci. 41, 1 (1942); J. Am. Chem. Soc. 64, 1712 (1942).

[6] E. A. DiMarzio, J. Chem. Phys. 35, 658 (1961).

[7] E. A. Guggenheim, Proc. R. Soc. London A 183, 203 (1944).

[8] A. Ghosh and D. Dhar, Europhys. Lett. 78, 20003 (2007).

[9] D. A. Matoz-Fernandez, D. H. Linares, and A. J. Ramirez-Pastor, Europhys. Lett. 82, 50007 (2008).

[10] D. A. Matoz-Fernandez, D. H. Linares, and A. J. Ramirez-Pastor, Physica A 387, 6513 (2008).

[11] D. A. Matoz-Fernandez, D. H. Linares, and A. J. Ramirez-Pastor, J. Chem. Phys. 128, 214902 (2008).

[12] P. Longone, D. H. Linares, and A. J. Ramirez-Pastor, Pap. Phys. 1, 010005 (2009).
[13] P. Longone, D. H. Linares, and A. J. Ramirez-Pastor, J. Chem. Phys. 132, 184701 (2010).

[14] D. H. Linares, F. Romá, and A. J. Ramirez-Pastor, J. Stat. Mech.: Theory Exp. (2008) P03013.

[15] D. A. Matoz-Fernandez, D. H. Linares, and A. J. Ramirez-Pastor, Langmuir 27, 2456 (2011).

[16] D. Dhar, R. Rajesh, and J. F. Stilck, Phys. Rev. E 84, 011140 (2011).

[17] M. Dávila, F. Romá, J. L. Riccardo, and A. J. Ramirez-Pastor, Surf. Sci. 603, 683 (2009).

[18] F. Romá, J. L. Riccardo, and A. J. Ramirez-Pastor, Langmuir 22, 3192 (2006).

[19] A. J. Ramirez-Pastor, J. L. Riccardo, and V. D. Pereyra, Langmuir 16, 10167 (2000).

[20] D. Nicholson and N. D. Parsonage, Computer Simulation and the Statistical Mechanics of Adsorption (Academic, London, 1982).

[21] D. P. Landau and K. Binder, A Guide to Monte Carlo Simulations in Statistical Physics (Cambridge University Press, Cambridge, UK, 2005). 
[22] N. Metropolis, A. W. Rosenbluth, M. N. Rosenbluth, A. H. Teller, and E. Teller, J. Chem. Phys. 21, 1087 (1953).

[23] J. P. Hansen and L. Verlet, Phys. Rev. 184, 151 (1969); K. Binder, J. Stat. Phys. 24, 69 (1981); Z. Phys. B 45, 61 (1981); T. L. Polgreen, Phys. Rev. B 29, 1468 (1984).

[24] Bethe-lattice calculations [16,25] could be useful to analyze this interesting issue.
[25] J. F. Stilck, K. D. Machado, and P. Serra, Phys. Rev. Lett. 76, 2734 (1996); J. F. Stilck, P. Serra, and K. D. Machado, ibid. 89, 169602 (2002); P. Serra, J. F. Stilck, W. L. Cavalcanti, and K. D. Machado, J. Phys. A 37, 8811 (2004); K. D. Machado, M. J. de Oliveira, and J. F. Stilck, Phys. Rev. E 64, 051810 (2001); D. P. Foster, J. Phys. A 40, 1963 (2007); T. J. Oliveira, J. F. Stilck, and P. Serra, Phys. Rev. E 80, 041804 (2009). 